\title{
Baseline platelet parameters for predicting early platelet response and clinical outcomes in patients with non-cardioembolic ischemic stroke treated with clopidogrel
}

\author{
Wenxian $\mathrm{Li}^{1, *}$, Xiaomei Xie ${ }^{1, *}$, Di Wei ${ }^{5, *}$, Shijun Zhang ${ }^{2}$, Yuanling Wu ${ }^{1}$, Xuejun Fu ${ }^{3}$, \\ Zhen Jing ${ }^{1}$, Weibiao Lu ${ }^{1}$, Xinqiang Lai ${ }^{4, \#}$ and Li'an Huang ${ }^{1, \#}$ \\ ${ }^{1}$ Department of Neurology, The First Affiliated Hospital, Jinan University, Guangzhou, Guangdong, 510632, P.R. China \\ ${ }^{2}$ Department of Neurology, People's Hospital of Zengcheng District, Guangzhou, Guangdong, 510180, P.R. China \\ ${ }^{3}$ Department of Neurology, People's Hospital, Second Clinical College, Jinan University, Shenzhen, 518020, P.R. China \\ ${ }^{4}$ Analysis and Testing Center, Jinan University, Guangzhou, Guangdong, 510632, P.R. China \\ ${ }^{5}$ Department of Urology, Xijing Hospital, Fourth Military Medical University, Xi'an, Shanxi, 710032, P.R. China \\ *These authors contributed equally to this work and share first authorship \\ \#These authors contributed equally to this study and share corresponding authorship \\ Correspondence to: Li'an Huang, email: huanglian1306@126.com \\ Xinqiang Lai, email: xinqianglai@126.com
}

Keywords: mean platelet volume, platelet count, clopidogrel resistance, non-cardioembolic ischemic stroke, platelet response Received: June 11, $2017 \quad$ Accepted: September 21, $2017 \quad$ Published: October 07, 2017

Copyright: Li et al. This is an open-access article distributed under the terms of the Creative Commons Attribution License 3.0 (CC BY 3.0), which permits unrestricted use, distribution, and reproduction in any medium, provided the original author and source are credited.

\section{ABSTRACT}

Purpose: The present study investigated whether routine baseline platelet parameters(BPPs) detected before clopidogrel therapy in acute non-cardioembolic ischemic stroke(NCIS) could predict early platelet response and future clinical outcomes.

Results: The CYP2C19 polymorphisms constituted independent risk factors for LCR. The number of female patients, the incidence of diabetes mellitus (DM), the level of low-density lipoprotein(LDL) cholesterol, and the neutrophil-to-lymphocyte ratio(NLR) were significantly high in the clinical clopidogrel resistance (CCR) group. However, none of the BPPs had a significant association with laboratory clopidogrel resistance (LCR) or discriminated with the cut-off values regarding LCR or CCR. The patients were divided into two groups according to the average mean platelet volume(MPV) or platelet count(PC). We found that the HbA1c level, the number of female patients, and the CCR were higher in the groups with elevated MPV ( $\geq 10.6 \mathrm{fL})$ and PC ( $\left.\geq 235 \times 10^{\circ} / \mathrm{L}\right)$; the LCR, the NIHSS score at discharge, and elevated MPV and PC were risk predictors for CCR.

Materials and Methods: This study included 196 patients with acute NCIS who underwent routine blood tests upon admission, were treated with clopidogrel, and were followed up for 6 months. Early platelet response was assessed and the CYP2C19 genetic variants were screened for. All participants were categorized into either laboratory clopidogrel resistance(LCR) or clinical clopidogrel resistance (CCR) groups.

Conclusions: Elevated baseline MPV and PC before clopidogrel therapy, as well as CYP2C19 gene variants, should be included in a risk algorithm for NCIS. Furthermore, other nongenetic clinical risk factors should be assessed for optimal prediction of the risk for thrombotic events because of individual variability in platelet response to clopidogrel.

\section{INTRODUCTION}

Platelets exert a central role in the development of ischemic stroke (IS) by the pathophysiology of thrombosis. The efficacy and safety of clopidogrel in preventing subsequent thrombotic events in non-cardioembolic ischemic stroke (NCIS) are well-established [1]. However, a considerable proportion of patients (prevalence 4-30\%) continue to experience recurrent ischemic thrombotic complications $[2,3]$. The platelet aggregation function 
cannot be inhibited by clopidogrel effectively. This phenomenon might be attributed to the individual variability in the platelet response to clopidogrel therapy and may be associated with a high incidence of recurrent thrombotic events or poor prognosis, arising because of clopidogrel resistance (CR). Currently, CR is further characterized by tests on platelet functions [3] and by the analysis of genetic polymorphisms [4]. The baseline platelet parameters (BPPs) can be obtained easily and quickly upon admission. Several studies have focused on BPPs within the normal range that have been related to risk factors for cardiovascular or cerebrovascular diseases and impaired antiplatelet therapy responses [5-12]. These included an elevated mean platelet volume (MPV) [11], a high platelet-large cell ratio (P-LCR) $[6,7,9,13]$, an increased platelet distribution width (PDW) [9], an elevated platelet count (PC) [14], and high plateletcrit (PCT) [15]. However, these theories remain controversial $[10,16]$. Thus, the pre-procedural predictors of the response to platelet inhibitors or CR from the perspective of cost-effectiveness, time-saving, and technical simplicity are in demand. The present study was designed to assess whether the routine baseline platelet indicators detected before clopidogrel therapy in acute NCIS can predict early platelet response and future clinical outcomes.

\section{RESULTS}

\section{Relevant demographic characteristics of the patients}

A total of 258 patients with NCIS fulfilled the inclusion and exclusion criteria during the study period. In total, 62 cases were excluded for the following reasons: seven patients were diagnosed with cardioembolic stroke, three patients had an undetermined etiology, 23 patients received other antiplatelet drugs after discharge, 21 did not undergo genetic analysis, and eight were lost to follow-up. Finally, 196 patients were available for further analysis. A flow chart of the current study is shown in Supplementary Figure 1. A total of $78(39.80 \%)$ patients had laboratory clopidogrel resistance (LCR) and 37 (18.88\%) patients had clinical clopidogrel resistance (CCR). Moreover, 14 patients had stroke progression during hospitalization, 17 patients developed recurrent strokes, and six patients had other ischemic vascular events during the 6-month followup period. The relevant demographic characteristics of the LCR or CCR groups are summarized in Table 1A and 1B.

The mean level of MPV was $10.6 \mathrm{fL}$. The patients were stratified into two groups according to the MPV value, namely, the low MPV group (MPV < $10.6 \mathrm{fL}$; $n=95)$ and the elevated MPV group (MPV $\geq 10.6 \mathrm{fL}$; $n=101)$. The mean level of PC was $235 \times 10^{9} / \mathrm{L}$. Patients were also stratified into two groups according to the $\mathrm{PC}$ value, namely, the low PC group $\left(\mathrm{PC}<235 \times 10^{9} / \mathrm{L}\right.$; $n=110)$ and the elevated $\mathrm{PC}$ group $\left(\mathrm{PC} \geq 235 \times 10^{9} / \mathrm{L}\right.$; $n=86$ ). The relevant demographic characteristics of the MPV and PC groups are presented in Table 2A and 2B, respectively.

\section{Associations of BPPs with platelet reactivity index (PRI) and gene polymorphisms}

\section{Associations of BPPs with PRI}

A strong positive correlation was observed between the MPV and the baseline PRI (BPRI) before clopidogrel treatment of acute NCIS ( $r=0.184, P<0.01$; Figure 1A). On the other hand, a weak, non-significant correlation was observed between MPV and the post-treatment PRI (PPRI) for 7 days; the other BPPs (PC, PDW, PCT, and P-LCR) did not correlate with BPRI or PPRI (all PS $>0.05)$.

\section{Associations of BPPs with gene polymorphisms}

In patients with two gene polymorphisms (CYP2C19 [636G $>A]$ ], CYP2C19 [681G $>A]$ ), the mean values of each of the BPPs (MPV, PC, PDW, PCT, and P-LCR) were similar in the variant genotype and the wild-type gene (all $P \mathrm{~s}>0.05)$. Moreover, the variant genotypes (GG/GA) and the wild-type gene (GG) associated with each gene polymorphism did not show any significant difference between the MPV (low and elevated) and the PC groups (low and elevated; all $P \mathrm{~s}>0.05$; Supplementary Table $1 \mathrm{~A}$ and 1B).

\section{Interaction of BPPs and non-genetic risk factors}

High MPV at the baseline was associated with a low PC before treatment $(\mathrm{r}=-0.413, P<0.0001$; Figure $1 \mathrm{~B})$, and the elevated MPV group had a low PC (214.23 \pm 48.92 vs. $256.64 \pm 65.21, P=0.015$; Table 2A).

Compared to those in the low MPV and PC groups, the number of female patients and hemoglobin A1c (HbA1c) levels were higher in the elevated MPV and $\mathrm{PC}$ groups (all $\mathrm{Ps}<0.05$; Table $2 \mathrm{~A}$ and $2 \mathrm{~B}$ ). However, no interaction effect was found between $\mathrm{MPV} / \mathrm{PC}$ and the neutrophil-to-lymphocyte ratio (NLR) (all $P_{\mathrm{S}}>0.05$; Table 2A and 2B).

\section{Associations of BPPs with LCR and CCR}

\section{Associations of BPPs with LCR}

In this study, all BPPs (MPV, PC, PDW, PCT, and P-LCR) were similar in the LCR group as compared to the non-LCR group (all $P_{\mathrm{S}}>0.05$; Table 1A).

\section{Associations of BPPs with CCR}

The incidence of the elevated MPV $(\geq 10.6 \mathrm{fL})$ and PC $\left(\geq 235 \times 10^{9} / \mathrm{L}\right)$ was significantly higher in the CCR group than in the non-CCR group (all $P \mathrm{~S}<0.005$ ). However, the other BPPs (PDW, PCT, and P-LCR) were similar (all $P_{\mathrm{S}}>0.05$; Table $2 \mathrm{~A}$ and $2 \mathrm{~B}$ ). 
Table 1A: Relevant demographic characteristics of the LCR and the non-LCR group

\begin{tabular}{|c|c|c|c|}
\hline Characteristics & $\operatorname{LCR}(n=78)$ & non-LCR $(n=118)$ & $P$ value \\
\hline \multicolumn{4}{|l|}{ Non-genetic risk factors } \\
\hline \multicolumn{4}{|l|}{ VRFs } \\
\hline Age (years) & $63.67 \pm 10.61$ & $62.65 \pm 11.60$ & 0.280 \\
\hline Men, $n(\%)$ & $55(70.51)$ & $81(68.64)$ & 0.781 \\
\hline Smoking history, $n(\%)$ & $30(38.46)$ & $51(43.22)$ & 0.508 \\
\hline Drinking history, $n(\%)$ & 17 (21.79) & $30(25.42)$ & 0.560 \\
\hline Hypertension, $n(\%)$ & $49(62.82)$ & $75(63.56)$ & 0.916 \\
\hline $\mathrm{DM}, n(\%)$ & $26(33.33)$ & $25(21.19)$ & 0.058 \\
\hline Dyslipidemia, $n(\%)$ & $8(10.26)$ & $16(13.56)$ & 0.490 \\
\hline $\mathrm{CHD}, n(\%)$ & $5(6.41)$ & $4(3.39)$ & 0.323 \\
\hline Stroke/TIA, $n(\%)$ & $8(10.26)$ & $14(11.86)$ & 0.727 \\
\hline \multicolumn{4}{|l|}{ BPPs } \\
\hline $\mathrm{PC}\left(\times 10^{9} / \mathrm{L}\right)$ & $235.73 \pm 59.41$ & $234.06 \pm 62.01$ & 0.749 \\
\hline MPV (fL) & $10.63 \pm 0.98$ & $10.50 \pm 0.89$ & 0.317 \\
\hline PDW (fl) & $12.86 \pm 2.12$ & $12.46 \pm 1.99$ & 0.562 \\
\hline PCT (\%) & $0.25 \pm 0.06$ & $0.24 \pm 0.06$ & 0.641 \\
\hline P-LCR (\%) & $30.19 \pm 7.79$ & $28.84 \pm 7.25$ & 0.527 \\
\hline \multicolumn{4}{|l|}{ Laboratory values } \\
\hline Cholesterol (mmol/L) & $4.91 \pm 1.22$ & $4.95 \pm 1.75$ & 0.758 \\
\hline Triglyceride (mmol/L) & $1.70 \pm 0.83$ & $1.83 \pm 1.40$ & 0.106 \\
\hline HDL-triglyceride (mmol/L) & $1.11 \pm 0.26$ & $1.17 \pm 0.51$ & 0.190 \\
\hline LDL-triglyceride (mmol/L) & $3.13 \pm 0.99$ & $3.05 \pm 0.99$ & 0.952 \\
\hline HbA1C (\%) & $6.89 \pm 2.31$ & $6.43 \pm 1.66$ & $0.010^{*}$ \\
\hline NLR (\%) & $3.25 \pm 3.08$ & $3.01 \pm 1.98$ & 0.319 \\
\hline \multicolumn{4}{|l|}{ Gene polymorphisms } \\
\hline CYP2C19 (636G>A) & & & $0.026^{*}$ \\
\hline GG & $51(65.38)$ & $94(79.66)$ & \\
\hline GA/AA & $27(34.62)$ & $24(20.34)$ & \\
\hline CYP2C19 $(681 \mathrm{G}>\mathrm{A})$ & & & $0.021^{*}$ \\
\hline GG & $38(48.72)$ & $77(65.25)$ & \\
\hline $\mathrm{GA} / \mathrm{AA}$ & $40(51.28)$ & $41(34.75)$ & \\
\hline
\end{tabular}

Table 1B: Relevant demographic characteristics of the CCR and the non-CCR group

\begin{tabular}{|c|c|c|c|}
\hline Characteristics & $\operatorname{CCR}(n=37)$ & non-CCR $(n=159)$ & $P$ value \\
\hline \multicolumn{4}{|l|}{ Non-genetic risk factors } \\
\hline \multicolumn{4}{|l|}{$V R F s$} \\
\hline Age (years) & $64.35 \pm 10.33$ & $62.61 \pm 11.52$ & 0.267 \\
\hline Female, $n(\%)$ & $11(29.73)$ & $24(15.09)$ & $0.036^{*}$ \\
\hline Smoking history, $n(\%)$ & $14(37.83)$ & $67(42.14)$ & 0.632 \\
\hline Drinking history, $n(\%)$ & $8(21.62)$ & $39(24.53)$ & 0.709 \\
\hline Hypertension, $n(\%)$ & $26(70.27)$ & $97(61.01)$ & 0.294 \\
\hline $\mathrm{DM}, n(\%)$ & $15(40.54)$ & $34(21.38)$ & $0.015^{*}$ \\
\hline Dyslipidemia, $n(\%)$ & $6(16.22)$ & $17(10.69)$ & 0.347 \\
\hline $\mathrm{CHD}, n(\%)$ & $3(8.11)$ & $6(3.77)$ & 0.257 \\
\hline Stroke/TIA, $n(\%)$ & $5(13.51)$ & $17(10.69)$ & 0.624 \\
\hline
\end{tabular}




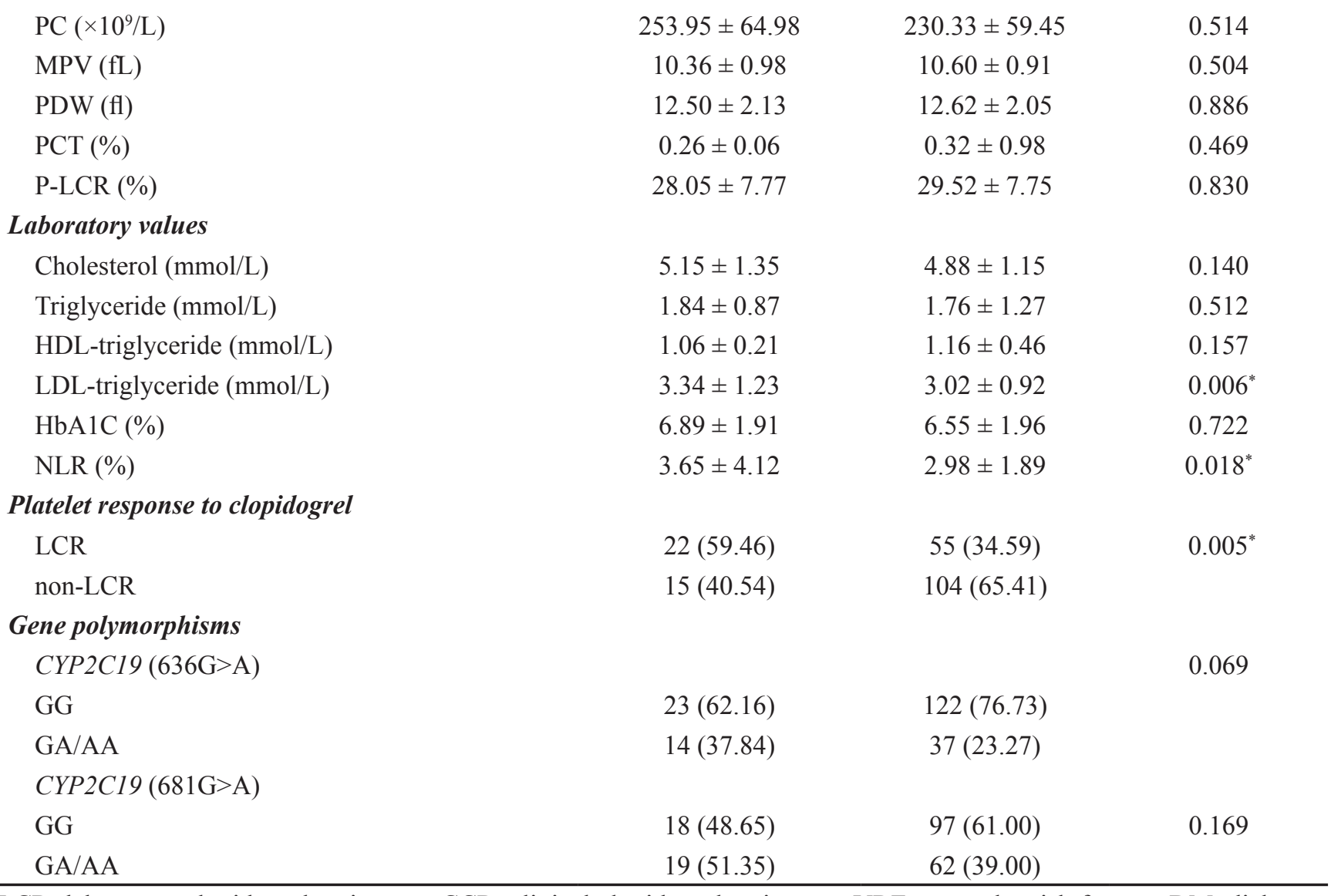

LCR, laboratory clopidogrel resistance; CCR, clinical clopidogrel resistance; VRFs, vascular risk factors; DM, diabetes mellitus; CHD, coronary heart disease; TIA, transient ischemic attack; BPPs, baseline platelet parameters; PC, platelet count; MPV, mean platelet volume; PDW, platelet distribution width; PCT, plateletocrit; P-LCR, platelet-large cell ratio; HDL, highdensity lipoprotein; LDL, low-density lipoprotein; GG, wild-type gene; GA/AA, variant genotypes.

Measurement data are expressed as a mean \pm standard deviation, and categorical data are expressed as $n(\%)$. ${ }^{*} P<0.05$.

In addition, receiver operating characteristic (ROC) analysis curve analysis was performed to determine the predictive cut-off value of MPV and PC in NCIS patients regarding LCR or CCR; however, no remarkable results were observed (Supplementary Figure 2). When the MPV and PC cut-off levels were set at $10.6 \mathrm{fL}$ and $235 \times$ $10^{9} / \mathrm{L}$, respectively, using the ROC curve, the sensitivity and specificity were $<60 \%$ for differentiating between the groups with LCR/CCR (area under the curve [AUC] $<0.7$; Figure 2).

\section{Risk predictors for LCR and CCR}

\section{Risk factors for LCR}

Compared with the wild-type gene (GG), the polymorphisms of the variant genotype (GA/AA)
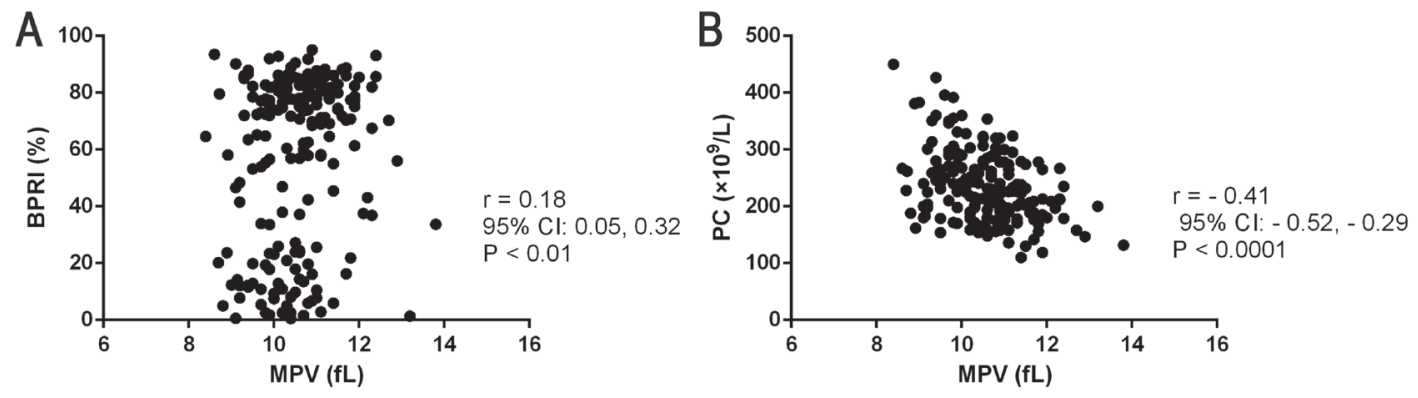

Figure 1: Associations between baseline BPPs and early platelet response to clopidogrel treatment for 7 days. Positive correlations between: (A) MPV and BPRI, (B) MPV and PC. 
of $C Y P 2 C 19(636 \mathrm{G}>\mathrm{A})\left(\chi^{2}=4.972, P=0.026\right)$ and CYP2C19 (681G $>$ A) $\left(\chi^{2}=5.296, P=0.021\right)$ were significantly associated with LCR (Table 1A). And these CYP2C19 genotypes were risk predictors for LCR $(C Y P 2 C 19$ [636G $>\mathrm{A}]$, odds ratio $[\mathrm{OR}]=2.894$, 95\% confidence interval $[\mathrm{CI}]: 1.427-5.552, P=0.003$; CYP2C19 (681G>A), OR = 2.654, 95\% CI: 1.401-5.027, $P=0.003$; Table 3A).

The BPRI values were significantly higher in the LCR group than in the non-LCR group $(70.62 \pm 21.43 \%$ vs. $47.59 \pm 31.33 \%$, respectively; $P<0.001)$. The HbA1c level was significantly higher in the LCR group than in the non-LCR group. Nevertheless, we did not find any other non-genetic risk factors, including vascular risk factors (VRFs) (data not shown). Moreover, none of the BPPs were associated with LCR (all PS $>0.05$; Table 1A).

In addition, the IS subtypes were not related to LCR (Supplementary Table 2A).

A
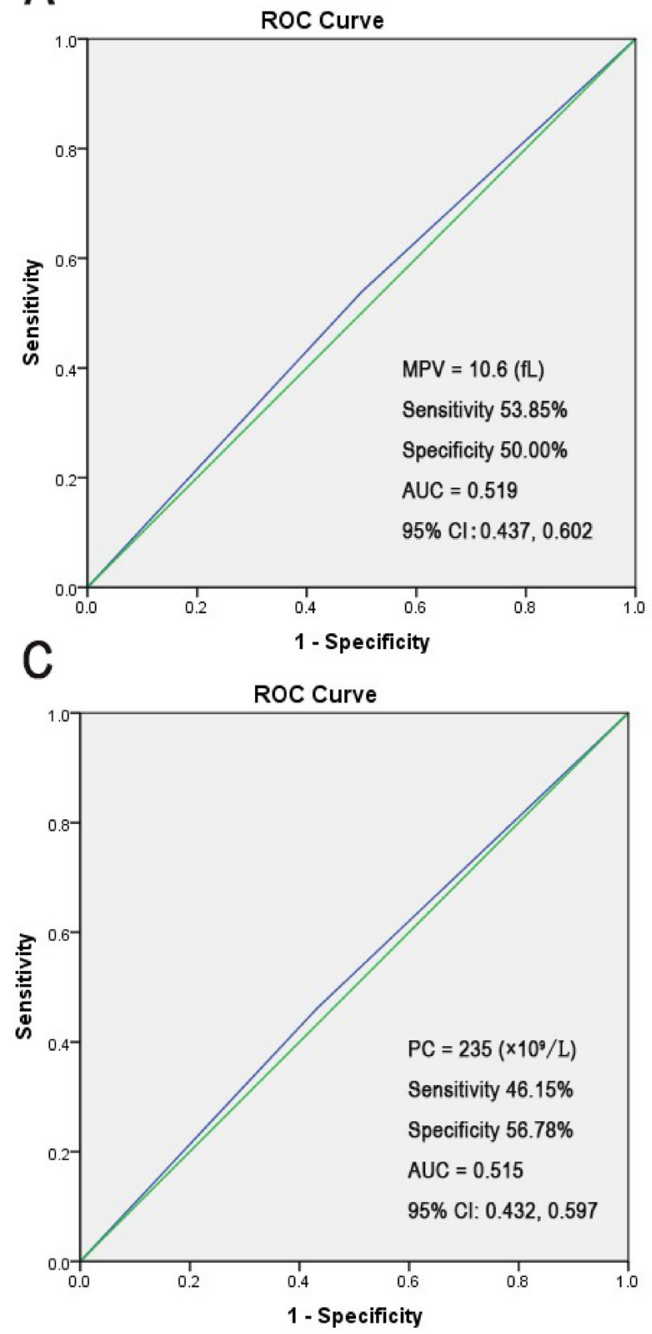

\section{Risk factors for CCR}

The CCR occurrence was significantly higher in the LCR group than in the non-LCR group $\left(\chi^{2}=7.782\right.$, $P=0.005$; Table 1B).

A large number of patients with CCR were female $\left(\chi^{2}=4.383, P=0.036\right)$; the CCR group had more patients with diabetes mellitus (DM) than did the non-CCR group $\left(\chi^{2}=5.875, P=0.015\right)$. In addition, the levels of lowdensity lipoprotein cholesterol (LDL-C) and NLR were significantly higher in patients with $\mathrm{CCR}$ than in the nonCCR group (CCR, $\mathrm{F}=7.588, P=0.006$; non-CCR, $\mathrm{F}=$ 5.699, $P=0.018$; Table 1B).

Non-conditional logistic regression analysis revealed that the risk predictors for CCR included elevated MPV $(\geq 10.6 \mathrm{fL} ; \mathrm{OR}=2.288,95 \% \mathrm{CI}: 1.088-4.814, \mathrm{P}$ $=0.029)$, elevated PC $\left(\geq 235 \times 10^{9} / \mathrm{L} ; \mathrm{OR}=2.177,95 \%\right.$ CI: $1.050-4.512, \mathrm{P}=0.036), \mathrm{LCR}(\mathrm{OR}=4.068,95 \% \mathrm{CI}$ :

B
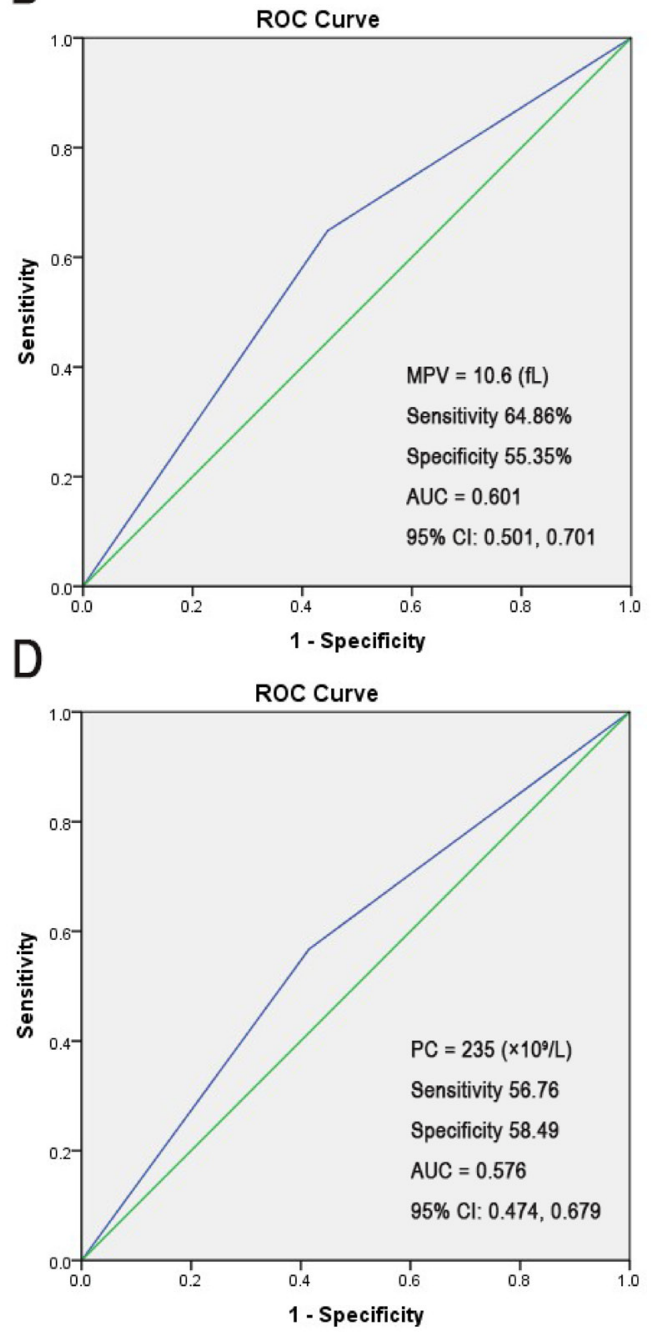

Figure 2: The ROC curve analysis of admission MPV/PC for predicting poor outcome (setting the cut-off value). (A) The MPV cut-off value was set at $10.6 \mathrm{fL}$ using the ROC analysis to predict LCR. (B) The MPV cut-off value was set at $10.6 \mathrm{fL}$ by using the ROC analysis to predict CCR. (C) The PC cut-off value was set at $235 \times 10^{9} / \mathrm{L}$ by using the ROC analysis to predict LCR. (D) The PC cut-off value was set at $235 \times 10^{9} / \mathrm{L}$ by using the ROC analysis to predict CCR. 
Table 2A: Relevant demographic characteristics of the MPV groups

\begin{tabular}{|c|c|c|c|}
\hline Characteristics & MPV $<10.6$ fL $(n=95)$ & $\mathrm{MPV} \geq 10.6 \mathrm{fL}(n=101)$ & $P$ value \\
\hline \multicolumn{4}{|l|}{ Non-genetic risk factors } \\
\hline \multicolumn{4}{|l|}{ VRFs } \\
\hline Age (years) & $63.06 \pm 11.39$ & $63.05 \pm 11.08$ & 0.945 \\
\hline Female, $n(\%)$ & $24(25.26)$ & $49(48.51)$ & $0.001^{*}$ \\
\hline Smoking history, $n(\%)$ & $44(46.32)$ & $37(36.63)$ & 0.169 \\
\hline Drinking history, $n(\%)$ & $28(29.47)$ & $19(18.81)$ & 0.081 \\
\hline Hypertension, $n(\%)$ & $55(57.89)$ & $69(68.32)$ & 0.130 \\
\hline $\mathrm{DM}, n(\%)$ & $27(28.42)$ & $24(23.76)$ & 0.458 \\
\hline Dyslipidemia, $n(\%)$ & $13(13.68)$ & $11(10.89)$ & 0.551 \\
\hline CHD, $n(\%)$ & $2(2.11)$ & $7(6.93)$ & 0.107 \\
\hline Stroke/TIA, $n(\%)$ & $11(11.58)$ & $11(10.89)$ & 0.879 \\
\hline \multicolumn{4}{|l|}{ BPPs } \\
\hline $\mathrm{PC}\left(\times 10^{9} / \mathrm{L}\right)$ & $256.64 \pm 65.21$ & $214.23 \pm 48.92$ & $0.015^{*}$ \\
\hline PDW (fl) & $11.15 \pm 1.48$ & $13.97 \pm 1.54$ & 0.253 \\
\hline РCТ (\%) & $0.25 \pm 0.06$ & $0.36 \pm 1.23$ & 0.120 \\
\hline P-LCR (\%) & $23.38 \pm 4.33$ & $34.77 \pm 6.01$ & 0.234 \\
\hline \multicolumn{4}{|l|}{ Laboratory values } \\
\hline Cholesterol (mmol/L) & $4.95 \pm 1.25$ & $4.92 \pm 1.13$ & 0.251 \\
\hline Triglyceride (mmol/L) & $1.81 \pm 1.44$ & $1.75 \pm 0.94$ & 0.384 \\
\hline HDL-triglyceride $(\mathrm{mmol} / \mathrm{L})$ & $1.13 \pm 0.26$ & $1.15 \pm 0.54$ & 0.210 \\
\hline LDL-triglyceride (mmol/L) & $3.08 \pm 1.03$ & $3.07 \pm 0.95$ & 0.316 \\
\hline $\operatorname{HbA1C}(\%)$ & $6.33 \pm 1.51$ & $6.81 \pm 2.22$ & $0.002^{*}$ \\
\hline NLR (\%) & $3.05 \pm 2.07$ & $3.16 \pm 2.81$ & 0.679 \\
\hline \multicolumn{4}{|l|}{$C R$} \\
\hline LCR & $41(43.16)$ & $37(36.63)$ & 0.351 \\
\hline $\mathrm{CCR}$ & $24(25.26)$ & $13(12.87)$ & $0.027^{*}$ \\
\hline
\end{tabular}

Table 2B: Relevant demographic characteristics of the PC groups

\begin{tabular}{|c|c|c|c|}
\hline Characteristics & $\mathrm{PC}<235 \times 10^{9} / \mathrm{L}(n=110)$ & $\mathrm{PC} \geq 235 \times 10^{9} / \mathrm{L}(n=86)$ & $P$ value \\
\hline \multicolumn{4}{|l|}{ Non-genetic risk factors } \\
\hline \multicolumn{4}{|l|}{ VRFs } \\
\hline Age (years) & $64.15 \pm 11.17$ & $61.65 \pm 11.15$ & 0.952 \\
\hline Female, $n(\%)$ & $24(21.82)$ & $36(41.86)$ & $0.003^{*}$ \\
\hline Smoking history, $n(\%)$ & $49(44.55)$ & $32(37.21)$ & 0.301 \\
\hline Drinking history, $n(\%)$ & $30(27.27)$ & $17(19.77)$ & 0.222 \\
\hline Hypertension, $n(\%)$ & $74(67.27)$ & $50(58.14)$ & 0.188 \\
\hline $\mathrm{DM}, n(\%)$ & $28(25.45)$ & $23(26.74)$ & 0.838 \\
\hline Dyslipidemia, $n(\%)$ & $10(9.09)$ & $14(16.28)$ & 0.128 \\
\hline CHD, $n(\%)$ & $6(5.45)$ & $3(3.49)$ & 0.514 \\
\hline Stroke/TIA, $n(\%)$ & $13(11.82)$ & $9(10.47)$ & 0.766 \\
\hline \multicolumn{4}{|l|}{ BPPs } \\
\hline MPV (fL) & $10.81 \pm 0.94$ & $10.23 \pm 0.80$ & 0.453 \\
\hline PDW (fl) & $13.23 \pm 2.07$ & $11.80 \pm 1.76$ & 0.283 \\
\hline
\end{tabular}




\begin{tabular}{|c|c|c|c|}
\hline PCT (\%) & $0.32 \pm 1.18$ & $0.29 \pm 0.04$ & 0.139 \\
\hline P-LCR (\%) & $30.40 \pm 7.79$ & $26.50 \pm 6.82$ & 0.849 \\
\hline \multicolumn{4}{|l|}{ Laboratory values } \\
\hline Cholesterol (mmol/L) & $4.78 \pm 1.12$ & $5.13 \pm 1.25$ & 0.485 \\
\hline Triglyceride (mmol/L) & $1.69 \pm 1.40$ & $1.89 \pm 0.90$ & 0.629 \\
\hline HDL-triglyceride $(\mathrm{mmol} / \mathrm{L})$ & $1.13 \pm 0.51$ & $1.16 \pm 0.27$ & 0.406 \\
\hline LDL-triglyceride $(\mathrm{mmol} / \mathrm{L})$ & $2.98 \pm 0.93$ & $3.20 \pm 1.05$ & 0.338 \\
\hline $\operatorname{HbA1C}(\%)$ & $6.48 \pm 1.77$ & $6.78 \pm 2.15$ & $0.044^{*}$ \\
\hline NLR (\%) & $3.35 \pm 2.87$ & $2.80 \pm 1.81$ & 0.128 \\
\hline \multicolumn{4}{|l|}{$C R$} \\
\hline LCR & $42(38.18)$ & $36(41.86)$ & 0.602 \\
\hline CCR & $15(13.64)$ & $22(25.58)$ & $0.034^{*}$ \\
\hline
\end{tabular}

LCR, laboratory clopidogrel resistance; CCR, clinical clopidogrel resistance; VRFs, vascular risk factors; DM, diabetes mellitus; CHD, coronary heart disease; TIA, transient ischemic attack; BPPs, baseline platelet parameters; PC, platelet count; MPV, mean platelet volume; PDW, platelet distribution width; PCT, plateletocrit; P-LCR, platelet-large cell ratio; HDL, highdensity lipoprotein; LDL, low-density lipoprotein; GG, wild-type gene; GA/AA, variant genotypes.

Measurement data are expressed as a mean \pm standard deviation, and categorical data are expressed as $n(\%)$. ${ }^{*} P<0.05$.

1.849-8.951, $P<0.001)$, and the National Institutes of Health Stroke Scale (NIHSS) score at discharge (OR $=$ $1.311,95 \%$ CI: $1.087-1.581, P=0.005$; Table 3B).

In addition, the IS subtypes, degree of intracranial stenosis, and infarction area were not related to CCR (Supplementary Tables 2B and 3).

\section{DISCUSSION}

\section{Definition of CR}

In the present study, CR, including LCR and CCR in conjunction, represents an accurate measure from a laboratory and clinical perspective. The average life span of the platelet is about 7 days; LCR is a laboratory parameter used for describing the early poor response to clopidogrel within 7 days. The vasodilator-stimulated phosphoprotein (VASP) phosphorylation assay is the most rational evaluation of ADP-induced platelet P2Y12 receptor activation and, as a consequence, a specific marker of the clopidogrel effect $[2,17]$. On the other hand, CCR represents the failure of clopidogrel treatment within 6 months, similar to adverse clinical outcomes. In addition, the carriers of CYP2C19 loss-of-function alleles are considered to be at great risk for IS if treated with clopidogrel, according to our previous studies $[2,4]$.

\section{Current clinical status of BPPs in patients with NCIS treated with clopidogrel}

Several studies have indicated that BPPs are simple and useful indicators, which can be collected from a plain and routine laboratory test. These BPPs are correlated with the development of adverse events or residual platelet reactivity under antiplatelet therapy in ischemic cardiovascular or cerebrovascular diseases $[5-7,9,11$, $12,14,18-20]$. However, several studies have indicated that BPPs are not associated with ischemic events $[8,10$, $16,21]$.

Thrombus formation and platelet activation play a pivotal role in the pathogenesis of acute IS. The estimations of platelet volume and number (which are both determinants of the platelet mass) are consistent with the function and activation of platelets [8]. The MPV is widely used to assess the platelet size and functions, while PC reflects the functions, production, and aging of platelets [14]. Furthermore, P-LCR is the percentage of platelets measuring $>12 \mathrm{fL}$ and is also a routinely assessed marker of platelet size and activity $[6,7,9,13]$. On the other hand, PDW indicates the morphometric indices of size distribution and is an index of platelet size heterogeneity; a large PDW can be an indicator of the prothrombotic status [22]. Notably, the MPV and PC are the two major indexes reflecting the platelets' functions and activities [14].

The main finding of the present study was that high baseline MPV $(\geq 10.6 \mathrm{fL})$ and PC $\left(\geq 235 \times 10^{9} / \mathrm{L}\right)$ were associated with CCR, exhibiting an inverse relationship with clopidogrel therapy in patients with NCIS: lowest (baseline MPV and PC level) in the non-CCR group and highest (baseline MPV and PC level) in the CCR group. The MPV has been shown to be an independent risk factor of IS in patients with atrial fibrillation [6, 23]; hence, patients with NCIS were selected as the primary research participants in the present study. An elevated MPV level might reflect an increase in reticulated or immature platelets. A high platelet turnover has been shown to be correlated with platelet aggregation and poor response to antiplatelet therapy. Large platelets exert a high metabolic 
Table 3A: Non-conditional logistic regression analysis of risk predictors for LCR

\begin{tabular}{lcccc}
\hline & Odds ratio & \multicolumn{2}{c}{ 95\% confidence interval } & \multirow{2}{*}{ P value } \\
\cline { 3 - 4 } & & Lower & Upper & \\
\hline CYP2C19 $(636 \mathrm{G}>\mathrm{A})$ & 2.894 & 1.427 & 5.552 & $0.003^{*}$ \\
CYP2C19 $(681 \mathrm{G}>\mathrm{A})$ & 2.654 & 1.401 & 5.027 & $0.003^{*}$ \\
\hline
\end{tabular}

LCR, laboratory clopidogrel resistance.

Input variables: VRFs (age, sex, smoking history, drinking history, hypertension, DM, dyslipidemia, CHD, stroke/ TIA); Gene polymorphisms [CYP2C19 (636G $>$ A; 681G $>$ A)]; BPPs (PC, MPV, PDW, PCT, P-LCR); Laboratory values (Cholesterol, Triglyceride, HDL-triglyceride, LDL-triglyceride, HbA1C, NLR); Clinical scores [(NIHSS, BI, mRS, Essen) at admission and at discharge].

${ }^{*} P<0.05$.

Table 3B: Non-conditional logistic regression analysis of risk predictors for CCR

\begin{tabular}{lcccc}
\hline & Odds ratio & \multicolumn{2}{c}{$\mathbf{9 5 \%}$ confidence interval } & \multirow{2}{*}{$\boldsymbol{P}$ value } \\
\cline { 3 - 3 } & & Lower & Upper & \\
\hline NCIS $(n=196)$ & & & & \multirow{2}{*}{$0.029^{*}$} \\
MPV $\geq 10.6$ & 2.288 & 1.088 & 4.814 & $0.036^{*}$ \\
PC $\geq 235$ & 2.177 & 1.050 & 4.512 & $0.005^{*}$ \\
NIHSS score at discharge & 1.311 & 1.087 & 1.581 & $<0.001^{*}$ \\
LCR & 4.068 & 1.849 & 8.951 & \\
\hline
\end{tabular}

Input variables: VRFs (age, sex, smoking history, drinking history, hypertension, DM, dyslipidemia, CHD, stroke/TIA); Gene polymorphisms [CYP2C19 (636G>A; 681G>A)]; BPPs (PC, MPV, PDW, PCT, P-LCR, MPV $\geq 10.6, \mathrm{PC} \geq 235$ ); Laboratory values (Cholesterol, Triglyceride, HDL-triglyceride, LDL-triglyceride, HbA1C, NLR); Clinical scores [(NIHSS, BI, mRS, Essen) at admission and at discharge]; LCR.

${ }^{*} P<0.05$.

activity and contain more high-density granules, produce more thromboxane B2, express a high level of P-selectin, fibrinogen, and glycoprotein IIb/IIIa receptors, and secrete large amounts of serotonin and $\beta$-thromboglobulin. Thus, the larger platelets are more prone to aggregation than the smaller platelets and easily form thrombi $[11,22,24]$. The patients with clinical CR showed a remarkable change in the positive MPV value than that in the clopidogrel responding patients $[23,25]$. Reportedly, the level of PC was lower in patients with ischemic cardiovascular diseases than in healthy controls [25]. However, different and conflicting results were presented in other studies [14, 26]. For example, the risk of cardiovascular disease was elevated at PC of $301 \times 10^{9} / \mathrm{L}-450 \times 10^{9} / \mathrm{L}$ [27]. Therefore, patients with high MPV and PC pre-treatment levels may lead to a predisposition towards the thrombogenic potential $[14,28]$.

Previous studies have established that the MPV and PC levels are inversely correlated [20]; the PC decreases with increasing MPV. However, the mechanism underlying this inverse relationship is still unclear. One study demonstrated that the stimulations resulting in an increase in PC and platelet volume during thrombopoiesis are regulated by independent mechanisms [22]. Herein, we indicated a positive correlation between MPV and BPRI, whereby a greater MPV was correlated with the expression of surface receptors, indicating an increased readiness of platelets to aggregate [9]. The BPRI reflects the aggregation function of platelets before clopidogrel treatment; a high BPRI might predict a high LCR rate in patients with NCIS [2].

\section{Predictive risk factors for CCR}

In the current study, we found that LCR was related only to CYP2C19 gene polymorphisms, which might be attributed to the mechanisms of clopidogrel underlying the CYP pathways on the antiplatelet response [3]. Elevated LDL-C levels and DM were correlated with CCR, which indicates that the adverse clinical outcomes of clopidogrel may be affected by VRFs. Moreover, VRFs exhibited a positive correlation with endothelial dysfunction and accelerated the development of atherosclerosis in a synergistic manner. On the other hand, LDL-C promoted atherosclerosis via endothelial damage and increased platelet aggregations [29]. The thromboinflammatory injury may be further aggravated in patients with high MPV, and endothelial cell damage following IS may be more likely to relapse into re-thrombosis in patients with high MPV because of increased thrombogenic potential and antiplatelet agent resistance [28]. The large platelets might promote the thrombotic events in a susceptible 
patient rather than simply being a consequence of the acute event [11]. With increasing MPV and total PC, the absolute number of aggregated platelets will also increase. Therefore, some BPPs putatively overwhelmed the other factors in determining the response to clopidogrel, as manifested by the elevated MPV and PC, among patients with NCIS, which contain excessive $\alpha$-granules and release prothrombotic substances; these substances aggravate the inflammation and endothelial dysfunction [7], affecting the future outcomes. We also found that the large number of female patients in the CCR, elevated MPV, and PC groups was not only because of the phenomenon that women with specific characteristics are associated with the occurrence of stroke [30] but also because it is unclear whether changes in endogenous estrogen levels can lead to altered platelet function. In addition, the high $\mathrm{HbA} 1 \mathrm{c}$ level might be related to LCR and increased MPV and PC; the increased levels of HbA1c positively correlated with increased platelet aggregation, turnover, and activation. We found that the NIHSS score at discharge was also a risk predictor for CCR, whereby a higher NIHSS score indicated a more severe neurological deficit. We also found that patients with LCR were more likely to develop $\mathrm{CCR}$, which is in line with our previous findings [2].

\section{The internal interaction of BPPs and non-genetic risk factors}

The PDW, PCT, and P-LCR did not correlate strongly with LCR and did not change in the CCR cases. Thus, a large variation in platelet size indicates a shift towards the population of bigger platelets.

In addition, MPV and PC were both found not to be discriminative regarding $\mathrm{LCR}$ or $\mathrm{CCR}$, according to the ROC curve analysis. We also verified if there was a correlation between NLR and MPV/PC, but no conclusion could be drawn. However, the NLR was significantly higher in patients with CCR than in the non-CCR group. One reason is that the role of BPPs in the prognosis of NCIS has not been elucidated. Atherosclerosis is a chronic and complex progressive inflammatory disease and not merely the passive accumulation of lipids within the arterial walls. Moreover, thrombogenesis and inflammation interact and reinforce each other in the pathogenesis of IS [8]. The platelet size is regulated by various intrinsic and extrinsic elements [8, 25]. Thus, the relationship between platelets and CCR might be a result of the complex influence of VRFs, and the inflammatory and therapeutic sensitivity. All these risk factors might conjointly contribute, at least partially, to the increased MPV and PC values in patients with NCIS with clopidogrel therapy. Moreover, higher MPV and PC values could also impact the adverse clinical outcomes conversely through these risk factors. Another potential explanation is that different methods were used to measure BPPs in different laboratories. The negative result may be partially explained by the insufficient exclusion criteria and different grouping methods.

A "one size fits all" approach to the antiplatelet treatment of NCIS is probably flawed. Targeted therapeutic changes to ease specifically the customization of antiplatelet therapy from the comprehensive perspective of BPPs, platelet functional tests, CYP2C19 gene variants, and other more non-genetic risk factors would likely provide a reasonable solution.

Nevertheless, the limitations of the current study should be mentioned. First, a potential bias due to the relatively small sample size and because no other gene polymorphisms were investigated cannot be excluded. Second, the PRI in the 6th month and the interaction of each BPP and VRF has not been evaluated. Third, the active metabolites of clopidogrel in various genotypes, as well as the plasma clopidogrel levels in NCIS, were not detected. Fourth, the recurrence rate of ischemic events may not be substantial owing to the short followup period. Fifth, the CCR was defined for all statistical analysis irrespective of the time variable. Therefore, additional multi-center clinical trials with larger samples are warranted to confirm our findings.

\section{MATERIALS AND METHODS}

\section{Ethical considerations}

The study was approved by the Medical Ethics Committee of the First Affiliated Hospital of Jinan University, China, and registered in the World Health Organization Clinical Trial Registry (registration number: ChiCTR-ONC-13003406). Written informed consent was obtained from each participant before enrollment.

\section{Study population}

Between February, 2012 and February, 2016, data of all consecutive patients within 3 days of acute NCIS onset receiving clopidogrel (Plavix ${ }^{\circledR}$ ) were prospectively collected and retrospectively analyzed at the First Affiliated Hospital of Jinan University, Department of Neurology. The clinical data were assimilated from hospital records by the attending physicians. After discharge, the clinical parameters were collected for 6 months by hospital visit or telephone. The scheduled telephonic followup was performed every month to address any queries, encourage compliance, and document any side-effects. The researchers evaluating the clinical events were blinded to the other data, such as genotypes. The patients with NCIS were treated and managed according to the relevant clinical guidelines [1]. Blood samples (4 mL) were obtained from the antecubital vein before and 7 days after clopidogrel $\left(\right.$ Plavix $^{\circledR}$ ) treatment for BPPs, gene polymorphisms, and early platelet response. A schematic of the current study is shown in Supplementary Figure 1. 


\section{Baseline platelet indicators}

All participants underwent routine blood tests for the measurements of BPPs (MPV, PC, PDW, PCT, and P-LCR) before antiplatelet therapy in the case of emergency or during admission. Blood samples were withdrawn with careful venipuncture from the antecubital vein, collected into ethylenediaminetetraacetic acid (EDTA) tubes, and preserved at room temperature. In this study, the BPPs were estimated within 2 hours of samplings. All measurements were processed on the XE5000 System (Sysmex Corporation, Japan).

The reference limits of each BPP were as follows: MPV [8.0-2.5 fL]; PC [100-300 × 10\%/L]; PDW [9.0-17.0 fl]; PCT [0.16-0.40\%]; and P-LCR [13-43\%].

\section{Definition of non-genetic risk factors}

The gathered non-genetic risk data included the patients' risk demographic characteristics, VRFs [31], clinical scores, administered drugs, and available laboratory data.

\section{The VRFs}

Hypertension was defined as a self-reported history or use of antihypertensive medication or a diagnosis of hypertension at discharge. DM was defined as a selfreported history or use of insulin or oral hypoglycemic treatment, glycosylated hemoglobin level $\geq 7 \%$, or a diagnosis of DM at discharge. Hyperlipidemia was defined as a self-reported history of use of lipid-lowering therapy or current treatment with lipid-lowering therapy, an LDL-C level of $2.6 \mathrm{mmol} / \mathrm{L}$ at admission, or a diagnosis of hyperlipidemia at discharge. A history of IS or transient ischemic attack was also defined. Coronary artery disease was defined as a reported history of myocardial infarction, angina pectoris, positive stress test, or cardiac surgery/ intervention. A current smoker was defined as a patient who smoked $\geq 1$ cigarette per day continuously for 6 months. A heavy drinker was defined as a patient who drank $>2$ units of alcohol per day on average (for male patients) or $>1$ unit of alcohol per day on average (for female patients).

\section{The clinical scores}

The neurological function of each patient was assessed using the NIHSS, Barthel Index (BI), Modified Rankin Scale (mRS), and Essen scores by the attending neurologists at admission and at discharge.

\section{The IS subtypes}

The patients with NCIS were classified into largeartery atherothrombosis (LAA) and cerebral small-vessel disease (cSVD) subtypes, according to the Chinese Ischemic Stroke Subclassification (CISS) criteria [32].

The infarction areas were categorized as anterior circulation, posterior circulation, and both. The degree of intracranial stenosis on magnetic resonance angiography or digital subtraction angiography was determined by the method of the WASID study [31], and categorized as mild, moderate, or severe.

\section{Definition of clopidogrel resistance}

\section{The LCR}

Flow cytometric measurements of the level of VASP phosphorylation was used for assessing the early platelet response to clopidogrel therapy [2]. The detection procedure of PRI using flow cytometry is shown in Figure 3. The PRI included the values at BPRI before clopidogrel treatment and at PPRI for 7 days. After obtaining the BPRI values, patients were administered a 300-mg oral loading dose of clopidogrel followed by a standard dosage of $75 \mathrm{mg}$ /day for at least 6 months. The PPRI values were obtained after 7 days. The assays were conducted by using a Platelet VASPFCM kit (Biocytex, Marseille, France) according to the manufacturer's instructions (Analysis and Testing Center, Jinan University).

The LCR was defined as PPRI $>50 \%$ [2, 33], which represented early poor response to clopidogrel.

\section{The CCR}

The CCR is the equivalent to adverse clinical outcomes, which were defined as the onset of progressive IS (an increase of NIHSS score $\geq 2$ ) during admission, recurrence of IS [34], or occurrence of other ischemic diseases within 6 months [2]. These events were confirmed by reviewing the hospital discharge reports. Alternatively, the data were collected by telephonic follow-up.

The IS recurrence was verified at the index hospitals based on the presence of new neurological deficits documented in the medical records combined with $\mathrm{CT}$ or MRI images [34]. At 3 and 6 months after discharge, the patients or their relatives were contacted over the telephone by trained research personnel at the First Affiliated Hospital of Jinan University and asked whether the patients had developed new symptoms or had been hospitalized again with another stroke. An experienced neurologist reviewed the patients' medical records to ensure a reliable diagnosis of IS recurrence. In patients with an unclear event who were not hospitalized, they would be adjudicated by a stroke neurologist and the principle investigator [35].

\section{Genetic analysis}

The polymorphisms of CYP2C19 gene loci within two genotypes, including CYP2C19 $(636 \mathrm{G}>\mathrm{A})$ and $C Y P 2 C 19(681 \mathrm{G}>\mathrm{A})$, were screened. The chain termination method was used to sequence the abovementioned genotypes (ABI 3730, Applied Biosystems Inc., Foster City, CA) [2], which were identified by the BGI Company (Beijing, China). 


\section{Statistical analysis}

All statistical analyses were performed using SPSS version 19.0A (IBM, Armonk, NY, USA). The measurement data were represented as the mean \pm standard deviation. The categorical data were presented as percentage. Groups were compared by independentsamples $t$-test. Pearson $\chi^{2}$ test and Fisher's exact test were used for assessing the categorical variables as appropriate. The Spearman's correlation coefficient was computed to examine the association between the two continuous variables. A $P$-value $<0.05$ was considered statistically significant. The ROC curve analysis determined the sensitivity and specificity with $95 \%$ CI for each BPP at the set cut-off values. At the set cut-off value, sensitivity and

A

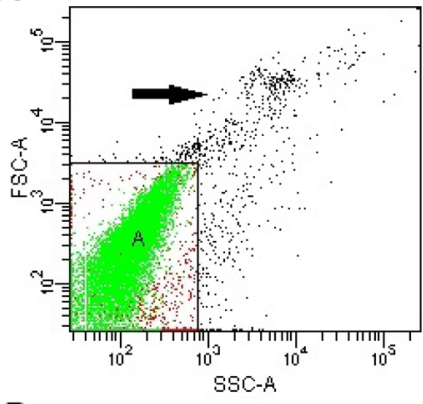

B

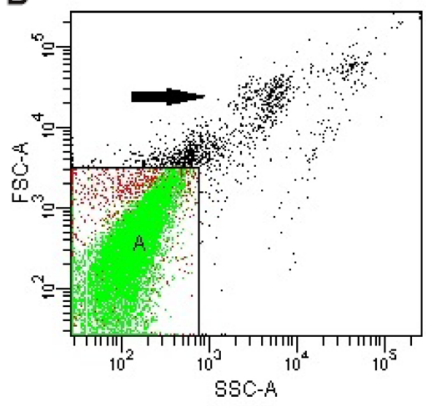

C

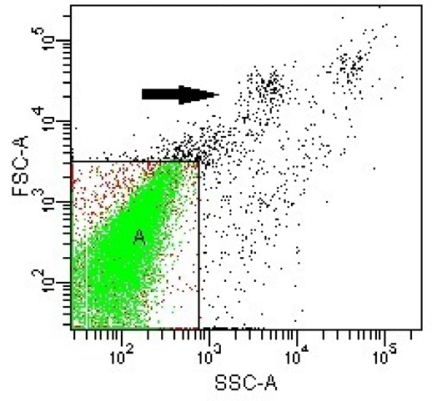

D

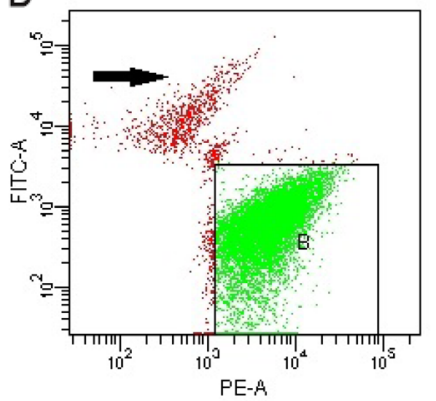

$\mathrm{E}$

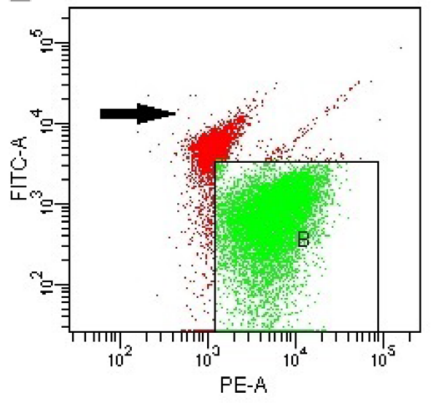

$\mathrm{F}$

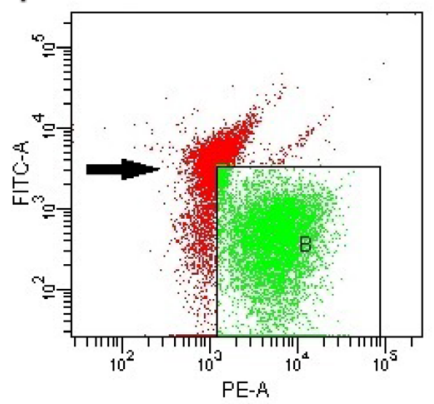

specificity over $60 \%$ and AUC over 0.7 were considered statistically significant.

Significant predictive risk factors for LCR and CCR were identified by non-conditional logistic regression analysis. The ORs and $95 \%$ CIs were calculated for each variable. The factors with a $P$-value $<0.10$ from the univariate analysis were entered into the model. Variables with $\mathrm{OR}>1$ and $P<0.05$ in the univariate analysis were included in the binary regression model as positive prediction risk factors.

\section{Abbreviations}

IS, ischemic stroke; NCIS, non-cardioembolic ischemic stroke; CR, clopidogrel resistance; BPPs, baseline

\section{G}

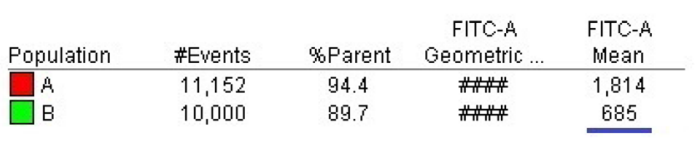

$\mathrm{H}$

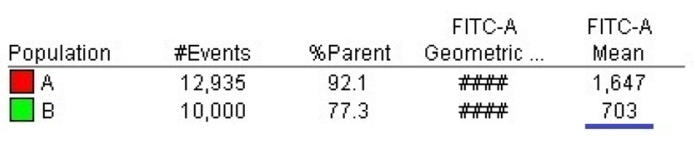

I

Figure 3: The detection procedure of PRI using flow cytometry. The T1, T2 and T3 tubes have the same position in regions A and B. The T1 and T2 tubes were experimental tubes and the T3 tubes were controls. (A-C) Setting region A of the T1, T2 and T3 tubes (white blood cells should be excluded from the region; black arrow indicates white blood cells), respectively. (D) Setting region B of the T1 tube (black arrow indicates the cell debris): a group of platelets that are activated by PGE1 and are shown in the combination of anti-VASP phosphorylated monoclonal antibodies. (E) Setting region B of the T2 tube (black arrow indicates the cell debris): the antibody combining with the granule group of the combination of ADP and P2Y12 and then activated by PGE1. (F) Setting region B of the T3 tube (black arrow indicates the cell debris): the negative control granule group of negative VASP phosphorylated antibody and PGE1/ADP combined with platelet. (G-I) Analysis of MFI of the T1, T2, and T3 tubes using CELL Quest software (The blue underline). 
platelet parameters; MPV, mean platelet volume; P-LCR, platelet-large cell ratio; PDW, platelet distribution width; PC, platelet count; PCT, plateletocrit; LCR, laboratory clopidogrel resistance; CCR, clinical clopidogrel resistance; BPRI, baseline platelet reactivity index; PPRI, post-treatment platelet reactivity index; $\mathrm{HbAlc}$, haemoglobin A1c; NLR, neutrophil to lymphocyte ratio; ROC analysis, Receiver operating characteristic analysis; AUC, area under the curve; VRFs, vascular risk factors; DM, diabetes mellitus; LDL-C, low-density lipoprotein cholesterol, NIHSS, National Institutes of Health Stroke Scale; VASP, vasodilator-stimulated phosphoprotein; EDTA, ethylenediaminetetraacetic acid; BI, Barthel Index; mRS, modified Rankin Scale; LAA, large-artery atherothrombosis; cSVD, cerebral small-vessel disease; PRI, platelet reactivity index; CI, confidence interval; ORs, odds ratios; CHD, coronary heart disease; TIA, transient ischemic attack; HDL, high-density lipoprotein.

\section{Author contributions}

Study conception and design: HLA LWX WD. Acquisition of data: LWX XXM WYL ZSJ. Data curation: XXM WYL JZ LWB. Analysis and interpretation of data: LWX XXM WD. Funding acquisition: HLA FXJ. Methodology: LWX WD LXQ. Writing-original draft: LWX. Writing-review \& editing: HLA LXQ FXJ.

\section{ACKNOWLEDGMENTS}

We are grateful to the clinicians and staffs at the first affiliated hospital of Jinan university who contributed to the blood samples and data collection for this study.

\section{CONFLICTS OF INTEREST}

There are no conflicts of interest.

\section{FUNDING}

This work was supported by the mechanism of Dl-3-n-butyphthalide treatment improves collateral circulation of focal acute cerebral ischemia in Beagles. The specific science-technology project of the prophylaxis and treatment of major diseases, China [NO. ZX01-C2016083]. The Project of public welfare research and capacity construction, Guangzhou, Guangdong [NO. 2017A020215200]. The Scientific and Technological Innovation Committee Research Topic of Shenzhen [NO. JCYJ20150403101028202]. The Construction Project of clinical and translational medical research of ischemic cerebrovascular diseases, Guangzhou [NO. 201508020004].

\section{REFERENCES}

1. Kernan WN, Ovbiagele B, Black HR, Bravata DM, Chimowitz MI, Ezekowitz MD, Fang MC, Fisher M, Furie KL, Heck DV, Johnston SC, Kasner SE, Kittner SJ, et al. Guidelines for the prevention of stroke in patients with stroke and transient ischemic attack: a guideline for healthcare professionals from the American Heart Association/American Stroke Association. Stroke. 2014; 45:2160-2236.

2. Zhang S, Lai X, Li W, Xiong Z, Xu A, Huang L. VASP phosphorylation and genetic polymorphism for clopidogrel resistance in Chinese patients with non-cardioembolic ischemic stroke. Thromb Res. 2014; 134:1272-1277.

3. Le Quellec S, Bordet JC, Negrier C, Dargaud Y. Comparison of current platelet functional tests for the assessment of aspirin and clopidogrel response. A review of the literature. Thromb Haemost. 2016; 116:638-650.

4. Pan Y, Chen W, Xu Y, Yi X, Han Y, Yang Q, Li X, Huang L, Johnston SC, Zhao X, Liu L, Zhang Q, Wang G, et al. Genetic Polymorphisms and Clopidogrel Efficacy for Acute Ischemic Stroke or Transient Ischemic Attack: A Systematic Review and Meta-Analysis. Circulation. 2017; 135:21-33.

5. Koh YY, Kim HH, Choi DH, Lee YM, Ki YJ, Kang SH, Park G, Chung JW, Chang KS, Hong SP. Relation between the Change in Mean Platelet Volume and Clopidogrel Resistance in Patients Undergoing Percutaneous Coronary Intervention. Curr Vasc Pharmacol. 2015; 13:687-693.

6. Choi SW, Choi DH, Kim HW, Ku YH, Ha SI, Park G. Clinical outcome prediction from mean platelet volume in patients undergoing percutaneous coronary intervention in Korean cohort: Implications of more simple and useful test than platelet function testing. Platelets. 2014; 25:322-327.

7. Li B, Liu X, Cao ZG, Li Y, Liu TM, Wang RT. Elevated mean platelet volume is associated with silent cerebral infarction. Intern Med J. 2014; 44:653-657.

8. Oz II, Yucel M, Bilici M, Serifoglu I, Sayin R, Ilikhan SU, Acikgoz M. Is Mean Platelet Volume a Reliable Marker to Predict Ischemic Stroke in the Follow-Up of Patients with Carotid Stenosis? J Stroke Cerebrovasc Dis. 2016; 25:404409.

9. Huczek Z, Filipiak KJ, Kochman J, Michalak M, Roik M, Piatkowski R, Grabowski M, Postula M, Opolski G. Baseline platelet size is increased in patients with acute coronary syndromes developing early stent thrombosis and predicts future residual platelet reactivity. A case-control study. Thromb Res. 2010; 125:406-412.

10. Pujol-Moix N, Vazquez-Santiago M, Morera A, Ziyatdinov A, Remacha A, Nomdedeu JF, Fontcuberta J, Soria JM, Souto JC. Genetic determinants of platelet large-cell ratio, immature platelet fraction, and other platelet-related phenotypes. Thromb Res. 2015; 136:361-366. 
11. Asher E, Fefer P, Shechter M, Beigel R, Varon D, Shenkman B, Savion N, Hod H, Matetzky S. Increased mean platelet volume is associated with non-responsiveness to clopidogrel. Thromb Haemost. 2014; 112:137-141.

12. Uzel H, Ozpelit E, Badak O, Akdeniz B, Baris N, Aytemiz F, Goldeli O. Diagnostic accuracy of mean platelet volume in prediction of clopidogrel resistance in patients with acute coronary syndrome. Anadolu Kardiyol Derg. 2014; 14:134-139.

13. Bakovic D, Pivac N, Eterovic D, Palada I, Valic Z, Paukovic-Sekulic B, Dujic Z. Changes in platelet size and spleen volume in response to selective and non-selective beta-adrenoceptor blockade in hypertensive patients. Clin Exp Pharmacol Physiol. 2009; 36:441-446.

14. Du J, Wang Q, He B, Liu P, Chen JY, Quan H, Ma X. Association of mean platelet volume and platelet count with the development and prognosis of ischemic and hemorrhagic stroke. Int J Lab Hematol. 2016; 38:233-239.

15. Guenancia C, Hachet O, Stamboul K, Bejot Y, Leclercq T, Garnier F, Yameogo NV, de Maistre E, Cottin Y, Lorgis L. Incremental predictive value of mean platelet volume/ platelet count ratio in in-hospital stroke after acute myocardial infarction. Platelets. 2017; 28:54-59.

16. Beyan C, Beyan E. Mean platelet volume may not be related to clopidogrel resistance in patients with acute coronary syndrome: Anadolu Kardiyol Derg. 2014; 14:405-6. https:// doi.org/10.5152/akd.2014.5552.

17. Montalescot G, Sideris G, Meuleman C, Bal-dit-Sollier C, Lellouche N, Steg PG, Slama M, Milleron O, Collet JP, Henry P, Beygui F, Drouet L. A randomized comparison of high clopidogrel loading doses in patients with non-STsegment elevation acute coronary syndromes: the ALBION (Assessment of the Best Loading Dose of Clopidogrel to Blunt Platelet Activation, Inflammation and Ongoing Necrosis) trial. J Am Coll Cardiol. 2006; 48:931-938.

18. Lai HM, Xu R, Yang YN, Ma YT, Li XM, Zhao Q, Chen QJ, Zhai H, Liu F, Chen BD. Association of mean platelet volume with angiographic thrombus burden and short-term mortality in patients with ST-segment elevation myocardial infarction undergoing primary percutaneous coronary intervention. Catheter Cardiovasc Interv. 2015; 1:724-733.

19. Arevalo-Lorido JC, Carretero-Gomez J, Alvarez-Oliva A, Gutierrez-Montano C, Fernandez-Recio JM, Najarro-Diez F. Mean platelet volume in acute phase of ischemic stroke, as predictor of mortality and functional outcome after 1 year. J Stroke Cerebrovasc Dis. 2013; 22:297-303.

20. Arikanoglu A, Yucel Y, Acar A, Cevik MU, Akil E, Varol S. The relationship of the mean platelet volume and C-reactive protein levels with mortality in ischemic stroke patients. Eur Rev Med Pharmacol Sci. 2013; 17:1774-1777.

21. Chen Y, Xiao Y, Lin Z, Xiao X, He C, Bihl JC, Zhao B, Ma X. The Role of Circulating Platelets Microparticles and Platelet Parameters in Acute Ischemic Stroke Patients. J Stroke Cerebrovasc Dis. 2015; 24:2313-2320.

22. Yilmaz F, Koklu E, Kizilirmak Yilmaz F, Sarionder Gencer E, Alparslan AS, Yildirimturk O. Evaluation of mean platelet volume and platelet distribution width in patients with asymptomatic intermediate carotid artery plaque. Kardiol Pol. 2017; 75:35-41.

23. Choi DH, Kang SH, Song H. Mean platelet volume: a potential biomarker of the risk and prognosis of heart disease. Korean J Intern Med. 2016; 31:1009-1017.

24. Tasdemir S, Erdem HB, Sahin I, Ozel L, Ozdemir G, Eroz R, Tatar A. Correlation with Platelet Parameters and Genetic Markers of Thrombophilia Panel (Factor II g.20210G >A, Factor V Leiden, MTHFR (C677T, A1298C), PAI-1, beta-Fibrinogen, Factor XIIIA (V34L), Glycoprotein IIIa (L33P)) in Ischemic Strokes. Neuromolecular Med. 2016; 18:170-176.

25. Quan W, Chen Z, Yang X, Li J, Li X, Weng Y, Li Y, Zhang $\mathrm{X}$. Mean platelet volume/platelet count ratio as a predictor of 90-day outcome in large artery atherosclerosis stroke patients. Int J Neurosci. 2017; 7:1-9.

26. Mueller C, Neumann FJ, Hochholzer W, Trenk D, Zeller T, Perruchoud AP, Buettner HJ. The impact of platelet count on mortality in unstable angina/non-ST-segment elevation myocardial infarction. Am Heart J. 2006; 151:e1-7.

27. Vinholt PJ, Hvas AM, Frederiksen H, Bathum L, Jorgensen MK, Nybo M. Platelet count is associated with cardiovascular disease, cancer and mortality: A populationbased cohort study. Thromb Res. 2016; 148:136-142.

28. Peng F, Zheng W, Li F, Wang J, Liu Z, Chen X, Xiao L, Sun W, Liu X. Elevated mean platelet volume is associated with poor outcome after mechanical thrombectomy. J Neurointerv Surg. 2017; 12:2016-012849.

29. de la Riva P, Zubikarai M, Sarasqueta C, Tainta M, Munoz-Lopetegui A, Andres-Marin N, Gonzalez F, Diez N, de Arce A, Bergareche A, Lopez de Munain A, Martinez-Zabaleta M. Nontraditional Lipid Variables Predict Recurrent Brain Ischemia in Embolic Stroke of Undetermined Source. J Stroke Cerebrovasc Dis. 2017; 13:30129-30125.

30. Bushnell C, McCullough LD, Awad IA, Chireau MV, Fedder WN, Furie KL, Howard VJ, Lichtman JH, Lisabeth LD, Pina IL, Reeves MJ, Rexrode KM, Saposnik G, et al. Guidelines for the prevention of stroke in women: a statement for healthcare professionals from the American Heart Association/American Stroke Association. Stroke. 2014; 45:1545-1588.

31. Wang Y, Zhao X, Liu L, Soo YO, Pu Y, Pan Y, Zou X, Leung TW, Cai Y, Bai Q, Wu Y, Wang C, Pan X, et al. Prevalence and outcomes of symptomatic intracranial large artery stenoses and occlusions in China: the Chinese Intracranial Atherosclerosis (CICAS) Study. Stroke. 2014; 45:663-669.

32. Gao S, Wang YJ, Xu AD, Li YS, Wang DZ. Chinese ischemic stroke subclassification. Front Neurol. 2011; 2:6.

33. Barragan P, Bouvier JL, Roquebert PO, Macaluso G, Commeau P, Comet B, Lafont A, Camoin L, Walter U, Eigenthaler M. Resistance to thienopyridines: clinical detection of coronary stent thrombosis by monitoring of 
vasodilator-stimulated phosphoprotein phosphorylation. Catheter Cardiovasc Interv. 2003; 59:295-302.

34. Ois A, Gomis M, Rodriguez-Campello A, CuadradoGodia E, Jimenez-Conde J, Pont-Sunyer C, Cuccurella G, Roquer J. Factors associated with a high risk of recurrence in patients with transient ischemic attack or minor stroke. Stroke. 2008; 39:1717-1721.
35. Zhang $\mathrm{C}$, Wang $\mathrm{Y}$, Zhao X, Liu L, Wang C, Pu Y, Zou X, Pan Y, Wong KS. Prediction of Recurrent Stroke or Transient Ischemic Attack After Noncardiogenic Posterior Circulation Ischemic Stroke. Stroke. 2017; 48:1835-1841. 